\title{
Co-creating quality: moving HE forwards in partnership with students
}

\author{
Sarah Dyer \\ University of Exeter, UK \\ Lisa Harris \\ University of Exeter, UK
}

Keywords: co-creation; student partnerships; culture change; online learning; Covid-19.

\section{The challenge}

The challenge we faced was how to engage students in dialogue about online and blended modules as they were being developed, rather than after they were delivered. The mechanisms for student feedback in existing quality assurance processes were not adequate for the emergency re-design of modules for the 2020/21 academic year. Quality assurance requires HE providers to engage students individually and collectively through mechanisms such as collecting feedback at module or programme level and working with course representatives and student unions. These processes are broadly retrospective, reviewing what has happened to inform what should happen next. They are also introspective, by which we mean that they look inward in their framing of quality and speak to - or reassure - internal HE audiences (universities and their regulators). In a time of crisis, we have seen that quality assurance has been less successful at reassuring those outside the sector, such as politicians, parents, or indeed students themselves, of the quality of education that universities are providing.

It was important to create forums for meaningful dialogue. We see meaningful dialogue as having empathy at its core, being willing and able to explore different perspectives, and being beneficial to all involved. We were particularly concerned about the broad-brush representations that were circulating in the media of online education as somehow compromised or impoverished (Dyer and Harris, 2020). The assumption that quality suffers when we teach online, meaning that we can neither provide good education, nor 
the sense of connection and community that made effective learning possible through more traditional methods, is not evidenced by our own experience or by research (see for example, the Innovating Pedagogy Report 2021, pp.36-39). We believe that a partnership approach to designing and assuring the quality of education provides an effective and constructive way to build trust in online and other novel or changed ways of doing things.

\section{The response}

The university has employed a group of 69 recent graduates as full-time members of staff (Digital Learning Developers, or DLDs) along with 119 current students (Digital Learning Assistants, or DLAs). Distributed across all six colleges, the DLDs are supporting teaching staff with the re-development of their modules for online learning, and the DLAs are providing feedback on the changes from a student perspective.

While the student voice has always been acknowledged, the level of investment in these roles has provided an essential bridge between academic staff, learning developers, and students. It has enabled the practice of having students as partners during what is becoming an extended period of significant change and uncertainty. Both DLDs and DLAs have current or very recent experience of being students and can provide that perspective in a far more direct and meaningful way than more traditional consultation through, for example, committee meetings or feedback surveys.

Recognising the importance of documenting the changes we've made, and to invite wider conversations, we set up a blog with our student DLAs as co-editors. Our objective was to help counteract the prevailing negativity around online learning by providing an evidencebased narrative of resilience, challenge, innovation, and opportunity from across the institution. However, we also found the process of educators and DLAs working side by side as editors fostered a deep and sustained partnership and mutual learning. We held weekly meetings to source new stories and track progress. The students have been able to take control and claim ownership through their roles as writers and editors, while applying their energy and enthusiasm as navigators and translators of experiences from the wider student body. As a result, they have gained employability skills, deepened their expertise, and extended their own digital presence. They have investigated what staff have 
found challenging about teaching online, what has been enjoyable, and how students are preparing for a very digital future in terms of work, life, and learning. They have commented on the ways in which their official roles build a sense of appreciation and being valued, and how leading by example and encouraging their peers to share their own stories can contribute to growing trust between educators and students.

\section{Recommendations}

A collaborative approach such as this, developing authentic student partnerships in the (re)design of education, can help us start to rebuild trust (Davidson and Katopodis, 2020). Universities must examine and innovate their mechanisms for assuring their quality of education. In the pandemic we have seen a breakdown of trust which damages both individual student learning and experience and the HE sector's social and political settlement. We recommend that quality assurance processes are designed to inform ongoing and future orientated questions and challenges and that they are designed to speak to real stakeholders (students, potential students, parents, and others). There are many models that could be adapted, such as citizens' juries.

We propose writing and editing as processes for building meaningful partnership between learning developers, educators, and students. These are activities which are authentic to the HE context. They create conditions for learning and are mutually beneficial to those involved. Through editing, students were able to direct attention to what they saw as meaningful. They evaluated and provided commentary. A blog is an inclusive forum for university students, any student who chooses is able to contribute, and all are able to read the contributions of others.

In conclusion the student voice embedded within the blog can be in the vanguard of the emerging new normal for education, rather than being applied retrospectively through more traditional quality assurance channels. By coordinating and communicating the stories of staff and students working in partnership we can collectively challenge the narrative of deficit and strive to replace it with one that speaks of resilience, trust, and authenticity. In turn this helps to build a more positive and forward looking narrative around the development of our educational priorities beyond the pandemic. 


\section{References}

Davidson, C. and Katopodis, C. (2020) 'Trust your students to be active participants in their learning', Times Higher Education, 26 June. Available at:

https://www.timeshighereducation.com/blog/trust-your-students-be-activeparticipants-their-learning (Accessed: 26 July 2021).

Dyer, S. and Harris, L. (2020) 'Let's take the remote out of online learning', WonkHE, 16 October. Available at: https://wonkhe.com/blogs/lets-take-the-remote-out-of-onlinelearning/ (Accessed: 26 July 2021).

Kukulska-Hulme, A., Bossu, C., Coughlan, T., Ferguson, R., FitzGerald, E., Gaved, M., Herodotou, C., Rienties, B., Sargent, J., Scanlon, E., Tang, J., Wang, Q., Whitelock, D. and Zhang, S. (2021) Innovating Pedagogy 2021: Open University Innovation Report, 9. Milton Keynes: The Open University.

\section{Author details}

Sarah Dyer is the director of the Exeter Education Incubator.

Lisa Harris is the director of digital learning at the University of Exeter Business School. 